www.uoc.edu/idp

RESEÑA

\title{
Webinar sobre la docencia en línea con RStudio Cloud
}

\author{
Jordi Mas Elias \\ Universitat Oberta de Catalunya
}

Fecha de publicación: octubre de 2020

La docencia a distancia en metodología y estadística presenta dificultades añadidas a las de otras asignaturas en línea, debido a que normalmente se requiere el uso de software de datos. El docente y el alumnado dedican buena parte del inicio del curso a la familiarización con un determinado programa estadístico y se destinan una gran cantidad de horas a resolver dudas y problemas de instalación, así como a comprender el lenguaje y las dinámicas del programa. Estos obstáculos se amplifican con la distancia física entre docente y alumnado, por lo que se ralentiza el aprendizaje.

Con el objetivo de facilitar la docencia en línea de asignaturas relacionadas con la metodología en ciencias sociales, el profesor de los Estudios de Derecho y Ciencia Política de la UOC Jordi Mas presentó el 20 de mayo el webinar titulado «RStudio Cloud en la docencia online en asignaturas de metodología y estadística» en el marco del ciclo Docencia no presencial de emergencia organizado por la universidad. La sesión se orientó a explicar el uso de la herramienta RStudio Cloud, diseñada por los creadores de RStudio, que ayuda en buena medida a solucionar estos problemas. Principalmente, se reducen las horas dedicadas a la instalación y familiarización, se eliminan los problemas particulares de cada alumno o alumna con el software y se incrementa el control del profesor sobre el escritorio de datos del alumnado. A principios de agosto de este año RStudio Cloud dejó de estar en versión beta. Con la primera versión algunas de sus ventajas han pasado a ser de pago. 


\title{
Cita recomendada
}

MAS ELIAS, Jordi (2020). «Webinar sobre la docencia en línea con RStudio Cloud». IDP. Revista de Internet, Derecho y Política, núm. 31. UOC [Fecha de consulta: dd/mm/aa] http://dx.doi.org/10.7238/idp.v0i31.3268

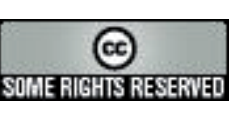

\begin{abstract}
Los textos publicados en esta revista están -si no se indica lo contrario- bajo una licencia Reconocimiento-Sin obras derivadas 3.0 España de Creative Commons. Puede copiarlos, distribuirlos y comunicarlos públicamente siempre que cite su autor y la revista y la institución que los publica (IDP. Revista de Internet, Derecho y Política; UOC); no haga con ellos obras derivadas. La licencia completa se puede consultar en: http://creativecommons.org/ licenses/by-nd/3.0/es/deed.es.
\end{abstract}

\section{Sobre el autor \\ Jordi Mas Elias \\ Universitat Oberta de Catalunya}

Es profesor de política internacional en la Universitat Oberta de Catalunya (UOC). En su etapa predoctoral ha estudiado en el Instituto de Barcelona de Estudios Internacionales, la London School of Economics y la Universidad Autónoma de Barcelona. Su investigación incluye regionalismo y economía política internacional. En docencia, imparte clases en el grado de Relaciones Internacionales y el máster de Conflict, Peace, and Security de la UOC en los ámbitos de análisis de datos, métodos de investigación y economía política internacional. 